\title{
Occipital gamma activation during Vipassana meditation
}

\author{
B. Rael Cahn $\cdot$ Arnaud Delorme $\cdot$ John Polich
}

Received: 27 October 2009/Accepted: 26 November 2009/Published online: 16 December 2009

(C) The Author(s) 2009. This article is published with open access at Springerlink.com

\begin{abstract}
Long-term Vipassana meditators sat in meditation vs. a control rest (mind-wandering) state for $21 \mathrm{~min}$ in a counterbalanced design with spontaneous EEG recorded. Meditation state dynamics were measured with spectral decomposition of the last $6 \mathrm{~min}$ of the eyes-closed silent meditation compared to control state. Meditation was associated with a decrease in frontal delta (1-4 Hz) power, especially pronounced in those participants not reporting drowsiness during meditation. Relative increase in frontal theta $(4-8 \mathrm{~Hz})$ power was observed during meditation, as well as significantly increased parieto-occipital gamma (35-45 Hz) power, but no other state effects were found for the theta $(4-8 \mathrm{~Hz})$, alpha $(8-12 \mathrm{~Hz})$, or beta $(12-25 \mathrm{~Hz})$ bands. Alpha power was sensitive to condition order, and more experienced meditators exhibited no tendency toward enhanced alpha during meditation relative to the control
\end{abstract}

B. R. Cahn $(\square)$

Division of Geriatric Psychiatry, Department of Psychiatry, University of California San Diego, 8950 Villa La Jolla Drive, Suite B-122, La Jolla, CA 92037, USA

e-mail: rael@ucsd.edu

\section{A. Delorme}

Institute for Neural Computation,

University of California San Diego,

La Jolla, CA, USA

A. Delorme

CERCO, CNRS, Universite Paul Sabatier,

133 Route de Narbonne, 31062 Toulouse Cedex 9, France

J. Polich

Cognitive Electrophysiology Laboratory,

Molecular and Integrative Neurosciences Department,

The Scripps Research Institute,

10550 North Torrey Pines Road,

La Jolla, CA 92037, USA

e-mail:polich@scripps.edu task. All participants tended to exhibit decreased alpha in association with reported drowsiness. Cross-experimental session occipital gamma power was the greatest in meditators with a daily practice of $10+$ years, and the meditation-related gamma power increase was similarly the strongest in such advanced practitioners. The findings suggest that long-term Vipassana meditation contributes to increased occipital gamma power related to long-term meditational expertise and enhanced sensory awareness.

Keywords Meditation - Electroencephalography (EEG) . Gamma $\cdot$ Mental state $\cdot$ Altered state of consciousness (ASC) · Vipassana

\section{Introduction}

The term meditation refers a set of diverse and specific methods of distinct attentional engagement, and recent reports have begun to focus specifically on state and trait measures of the same. Although the general effects of meditation on neuroelectric brain measures are still being characterized, consensus has emerged that coherence and/ or power for lower frequency spontaneous electroencephalographic (EEG) activity is enhanced as both a trait and a state effect in many forms of meditative practice (Cahn and Polich 2006).

Early studies of meditators implicated alpha $(8-12 \mathrm{~Hz})$ power increases as both a state and trait effect of Yogic, Zen, and Transcendental Meditation practice (Anand et al. 1961a; Kasamatsu and Hirai 1966; Kasamatsu et al. 1957; Wallace 1970; Wenger and Bagchi 1961). Later studies have failed to replicate the early findings of increased alpha in advanced practitioners but have reported increased alpha coherence, especially in assays of TM practitioners 
(Gaylord et al. 1989; Travis 1991; Travis and Pearson 1999; Travis et al. 2002), theta (4-8 Hz) power, especially in the assays of concentrative/focused attention practitioners (Aftanas and Golocheikine 2001; Baijal and Srinivasan 2009; Hebert and Lehmann 1977; Pan et al. 1994), or gamma effects (Lehmann et al. 2001; Lutz et al. 2004). Recent reports using LORETA to analyze EEG from Zen (Faber et al. 2008) and Qi-Gong (Tei et al. 2009) meditators further suggest that meditation may be associated with trait increased frontal delta activity, possibly indexing baseline relative inhibition of cognitive engagement and greater detachment from ongoing daily experience. Gamma findings have been reported as either distinguishing between various meditative states in an advanced practitioner (Lehmann et al. 2001), or as a state and trait marker for meditation in advanced Tibetan Buddhist practitioners engaging in compassion meditation (Lutz et al. 2004). One likely contributing factor to the inconsistency across studies is the lack of standardization with respect to meditative style, assessment methodology, and consideration of state effects for beginning vs. short- vs. long-term meditators (Cahn and Polich 2006). Toward this end, the present study was designed to provide fundamental information using EEG measures during meditation compared to control state effects in long-term Vipassana meditators.

\section{Vipassana meditation and present study}

Vipassana meditation is a Buddhist practice that involves focusing on present-moment sensory awareness with an equanimous and non-reactive mental set (Gunaratana 2002; Hart 1987). This tradition has served as the foundation for contemporary "mindfulness" meditation techniques such as the widely practiced mindfulness-based stress reduction (MBSR) currently used for clinical interventions (Davidson 2003; Grossman et al. 2004; Kabat-Zinn 1982, 2003). Development of greater awareness of and concomitant nonreactivity to interoceptive and exteroceptive sensory stimuli during formal Vipassana/mindfulness meditation is hypothesized to enhance self-awareness such that selective adaptive responding is facilitated at the expense of automated non-adaptive reactions, thereby promoting more successful management of stressful life situations (Hart 1987; Kabat-Zinn 2003; Lutz et al. 2007; Segal et al. 2002).

Vipassana practitioners of the Theravadan Vipassana tradition were assayed in the present study, and the majority had been taught in the tradition of Goenka (Hart 1987). This practice emphasizes attentional absorption in subtle somatosensory awareness and associated open monitoring without mental or emotional reactivity to such sensory experience. The specific Vipassana meditative technique involves attentional scanning of sensations throughout the body in an iterative and cyclic fashion, scanning body sensations from the top of the head to the toes and back again repeatedly, with the concomitant adoption of an attitude of detached observation and nonreactivity to any sensations and thoughts that may arise.

Systematic evaluation of meditation state in comparison with non-meditative thought conditions requires control cognitive tasks, and this condition was implemented with the instruction to let the mind wander freely through non-emotional thoughts and memories. This state was also chosen to mimic the aspects of the mind-wandering "default mode" state thought to have high ecological validity to a common mode of cognitive engagement in normal everyday life (Christoff et al. 2009; Smallwood and Schooler 2006).

Given that Vipassana meditation practice is thought to enhance the awareness of internal and external stimuli while reducing automated reactivity, it was hypothesized that increased frontal theta and alpha would be observed during meditation-i.e., reflecting increased purposeful attentional engagement and mental quiescence. Based on the view that Vipassana practice may promote an enhancement of sensory awareness through increased attentional engagement, we hypothesized that increased gamma activity would also be observed in meditation relative to the control condition, possibly in bilateral centroparietal and/or frontal areas, related to enhanced processing in frontal and somatosensory cortices. Recording conditions were designed to capture the time period when the meditation state was deep and stable and therefore most likely to contrast with the control state.

\section{Methods}

\section{Participants}

A total of $N=16$ Vipassana meditators $(F=5, M=11)$ were assessed $(M=45.5, \mathrm{SD}=9.8,24-56$ years $)$. As a group, these individuals had been meditating for a considerable period of time $(M=20.0, \mathrm{SD}=12.1,2.5$ 40 years), and all had been meditating daily (7 days/week) for at least 1 year $(M=13.0, \mathrm{SD}=10.7,1-30$ years $)$, with at least $0.5+\mathrm{h}$ or more each day $(M=1.3, \mathrm{SD}=0.7$, 0.5-3 h). Participants were recruited from a local Vipassana meditation community through word of mouth and e-mail and compensated $\$ 40$ for the 3-h study.

Recording conditions

EEG data were collected using a 19-channel ECI electrode cap from the following locations: Fp1, Fp2, F3, F4, F7, F8, $\mathrm{Fz}, \mathrm{C} 3, \mathrm{C} 4, \mathrm{~T} 7, \mathrm{~T} 8, \mathrm{Cz}, \mathrm{P} 3, \mathrm{P} 4, \mathrm{P} 7, \mathrm{P} 8, \mathrm{Pz}, \mathrm{O} 1$, and $\mathrm{O} 2$. These scalp locations were referenced to linked earlobes, with the ground at the forehead. Eye-movement (EOG) 
activity was assessed with electrodes placed at the outer canthi and above/below the left eye in line with the pupil for horizontal and vertical EOG monitoring using bipolar reference. Impedances were kept below $10 \mathrm{k} \Omega$. The signals were recorded with a band pass of $0.01-70 \mathrm{~Hz}(6 \mathrm{~dB}$ octave/slope) and digitization rate of $256 \mathrm{~Hz}$.

\section{Procedure}

The participants were instructed to sit on cushions and meditate within the Theravadan Vipassana meditation tradition or engage in the control neutral thinking state, with the order of the tasks counterbalanced across individuals. Participants were instructed to sit in the same posture for both the meditation and control task periods of recording and were fitted with headphones at the outset of the recording session that they wore throughout the recording. Pilot testing indicated that some participants found it difficult to refrain from engaging in their meditative practice when sitting in the meditative posture with eyes-closed. Participants were therefore told to think about emotionally neutral past events if they noticed themselves slipping into meditative practice state, otherwise to let their mind wander freely through non-emotional neutral thoughts. This control cognitive engagement was chosen to emulate a "mind-wandering" state with high ecological validity that can be contrasted with the purposeful attentional engagement of the meditation state (Christoff et al. 2009; Smallwood and Schooler 2006). Participants were informed that after $21 \mathrm{~min}$ of eyes-closed meditation or control thinking they would hear a series of tones over the headphones and that they were to simply continue their meditation or control cognitive engagement. Both passive and active presentations of simple tone stimuli were collected, with those data reported elsewhere (Cahn and Polich 2009).

At the conclusion of the first recording period, the participants were given the opportunity to stand and stretch before taking the same posture and seating position for the second recording of equal length. Immediately after each of the two recordings participants completed a short form indicating whether they experienced drowsiness or sleep onset during the experimental recording session and rating the depth of meditative experience on a 1-10 scale, with 1 indicating the normal waking and 10 indicating the deepest meditative absorption ever experienced.

\section{EEG analysis}

We focused the present analysis on the last 6 min of data from the $21 \mathrm{~min}$ recording period so as to assess the meditative state achieved after allowing an adequate period of time for the participants to fully absorb themselves into the meditation state. Given that the spontaneous brain rhythms generated during prolonged periods of eyes-closed rest tend to fluctuate and vary with factors such as reduced arousal and drowsiness, we did not average across the whole 21 min epoch. Future planned analysis will focus on the temporal evolution of brain dynamics in the meditation vs. control state so as to assess the onset vs. maintenance of meditation state effects and the relative stability of the spectral power dynamics across the two states. The last 6 min of EEG data from each of the two 21 min recording periods were first visually inspected, and transient muscleand movement-related artifacts were removed. The data were subsequently high-pass filtered at $0.5 \mathrm{~Hz}$ using FIR filter (Rabiner and Gold 1975). The extended ICA algorithm was then run on the data using the runica algorithm implemented within EEGLAB running on Matlab (Delorme and Makeig 2004; Delorme et al. 2007). The resultant independent components accounting for horizontal and vertical eye movements were then marked and removed from the data (Jung et al. 2000a, b) as detailed in the next section.

After removal of eye-movement-related artifact, data were segmented into non-overlapping 2-s artifact-free epochs. For the meditation and control states, means of $170.4 \pm 13.8$ and $164.1 \pm 26.6$ epochs were obtained. The Thomson multi-taper spectrum estimator (Matlab PMTM functions using time-bandwidth product of 4 and FFT length of 512) was applied to the cleaned continuous data for spectral decomposition (Thomson 1982). The output power values in $\mu \mathrm{V}^{2}$ were then log-transformed to $\mathrm{dB}$ units using $10 \times \log _{10}\left(\mu \mathrm{V}^{2}\right)$ formula to normalize the power value distributions.

Statistical analyses of the spectral power data were first applied to signals from all scalp channels using the bootstrap method (Wilcox 2005) and using false discovery rate (FDR) correction for multiple comparisons (Benjamini and Yekutieli 2001). Statistical assessments were conducted using analyses of variance with the factors of state (meditation vs. control) and electrode in the a priori regions of interest (midline electrodes for all frequencies, occipital electrodes for alpha). ANOVAs also were conducted on the frontal electrodes (F3 and F4) for delta and the occipital electrodes ( $\mathrm{O} 1$ and $\mathrm{O} 2)$ for gamma activity, as these were regions of statistical significance between states identified by the initial bootstrap statistical testing using FDR correction for the scalp data (see Fig. 2). Greenhouse-Geisser corrections were applied to the degree of freedom $(d f)$ to correct for violations of the sphericity assumption, and Tukey post hoc means comparisons were used to decompose reliable interactions.

Covariate ANOVAs were conducted using subject variables related to the order of engaging in meditation and control states, relative meditative expertise, and selfreports of drowsiness and meditative depth during 
experimental conditions. Bootstrap statistics and scalp map plotting were performed using the EEGLAB Matlab software (Delorme and Makeig 2004; Delorme et al. 2007) and custom Matlab scripts. Parametric statistics were run using the Statistica software and EEGLAB (Delorme and Makeig 2004; Delorme et al. 2007). The use of bootstrap statistics was employed as it allows for more robust statistical inference than standard parametric statistics since no assumption is made about the probability distribution at the population level (Wilcox 2005). However, studying complex patterns of covariate interaction is not yet available using bootstrap procedures in common statistical software, so that parametric statistics were employed.

\section{Independent component analysis (ICA)}

In addition to the statistical assessments, a parallel analysis using independent component analysis (ICA) of three classes of independent components from each subject was conducted: (1) eye-movement artifact, (2) temporal muscle artifact, and (3) occipital alpha power. For each subject, vertical eye-movement-related components were selected based on their characteristic scalp projection and a smooth exponentially decreasing spectrum (Delorme et al. 2007; Jung et al. 2000a). This approach was employed by identifying the EOG artifacts by simultaneously examining the eye channels (VEOG and HEOG) for activity to verify that the components were active only during those time periods when eye channels indicated eye movement. Muscle components produce component topographies with focal activities over specific channels, typically located temporally (T7 and T8), with a characteristic spectral signature containing strong spectral power over $20-30 \mathrm{~Hz}$ and an erratic spectrum (Jung et al. 2000a). Alpha occipital components were based on the $8-12 \mathrm{~Hz}$ frequency peak in the occipital areas, which was also associated with an additional peak at $20 \mathrm{~Hz}$. These analyses are illustrated in the following paragraphs.

The spectrum for the activity of these components was then computed on the independent components during both the meditation and the control periods with the same Thomson multi-taper spectrum estimator used on the channel data (Thomson 1982). Traditional $t$-tests were computed with bootstrap statistics with FDR correction for multiple comparisons, which were conducted between the power outcomes in the meditation vs. control states for each of the three classes of independent components at all frequencies (including gamma). This approach helped to assess whether eye or muscle gamma activity was greater in meditation state and whether the occipital alpha independent components replicated the occipital scalp channel gamma effect.

\section{Results}

Participants and self-report scales

The "depth of meditative state" from the (1-10) self-report scale indicated that the mean meditative depth experienced during the rest state was $1.7 \pm 1.4$, and meditative depth experience during the meditative state was $4.5 \pm 1.4(t$ test, $d f=15, P=0.00004)$. Drowsiness was reported by 7 of the 16 participants during the meditation and 10 of the 16 during the control thought condition. There was no reliable correlation between the self-reported depth of meditative state and either the number of years of daily practice $(r=0.24, P=0.36)$ or the number of hours of daily practice $(r=-0.02, P=0.93)$. A reliable correlation was obtained between the order of experimental session and the self-reported experience of drowsiness during the control state wherein those individuals meditating first were less likely to experience drowsiness during the control state $(r=0.52, P=0.041)$. No similar correlation was obtained between session order and drowsiness during meditation $(r=0.38, P=0.15)$ (see also Cahn and Polich 2009).

A negative correlation between the number of years of daily practice and reported drowsiness during the control state $(r=-0.60, P=0.015)$ was found, but not between number of years of daily practice and reported drowsiness during meditation $(r=-0.006, P=0.98)$. These findings imply that individuals with more years of daily meditation practices were less likely to report drowsiness specifically during the control cognitive condition. A negative correlation between current number of hours of daily practice reported and drowsiness during meditative state also was obtained $(r=-0.59, P=0.016)$. No association was observed for the control state drowsiness and number of hours of daily practice $(r=0.02, P=0.93)$, implying that greater current number of hours of current daily practice predicted decreased drowsiness specifically during meditation.

\section{EEG}

Figure 1 illustrates the mean amplitude spectral data averaged across meditation and control thought states with grand average scalp maps for each of the major bands represented for delta $(1-4 \mathrm{~Hz})$, theta $(4-8 \mathrm{~Hz})$, alpha $(8$ $12 \mathrm{~Hz}$ ), beta $(12-25 \mathrm{~Hz})$, and gamma $(35-45 \mathrm{~Hz})$. Preliminary analyses across all electrodes using bootstrap statistics and FDR correction for multiple comparisons indicated significant meditation state effects for delta and gamma log spectral power, but no such effects for theta, alpha, and beta power. Scalp maps for the two states are thus only shown separately for delta and gamma bands, and 


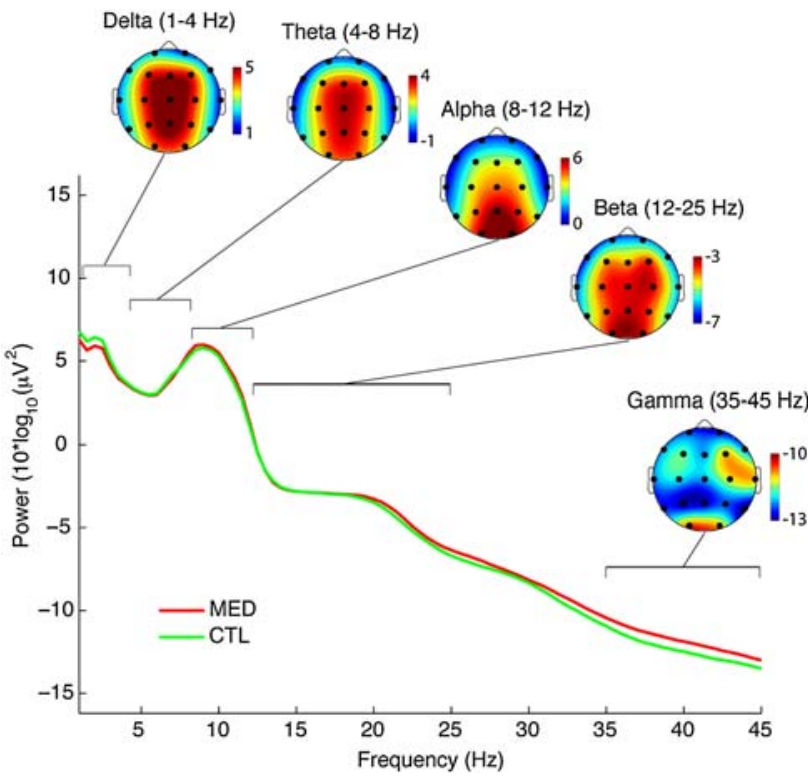

Fig. 1 The power spectrum for electrode $\mathrm{Cz}$ is displayed for both meditation and control states. Grand average scalp maps across states are displayed for each of the major frequency bands so as to indicate scalp topography of the various frequencies

Fig. 2 shows the spectral power grand average scalp maps for meditation and control states separately at both frequencies. Also indicated is a scalp map of the statistical significance for the comparison between the log spectral power at each electrode across the two states (all red electrode locations indicate significance at $P<0.05$ via bootstrap with FDR correction). Delta decreases were in bilateral frontal regions, and gamma increases were in parieto-occipital areas only (see Fig. 2).

Delta $(1-4 \mathrm{~Hz})$. As mentioned above, bootstrap analysis with FDR corrections for multiple comparisons analysis of the sensor data indicated decreased delta power during meditation relative to control period at bilateral frontal electrodes (F3, F4, F7, F8, C3, and C4, all $P<0.05)$. Parametric repeated-measures ANOVA analysis of the data was carried out as well, indicating that delta power at midline electrodes yielded no state $(F(1,15)=1.84$, $P=0.19)$, electrode $(F(2,30)=1.0, P=0.37)$, or interaction $(F(2,30)=0.01, \quad P=0.99) \quad$ effects, although somewhat greater delta power in control than meditation state was observed $(P=0.19)$. Frontal delta power at electrodes F3 and F4 was analyzed separately as delta activity at these electrodes was shown to be most significantly decreased in the bootstrap analysis of the scalp data. ANOVA analysis of the delta power at F3 and F4 demonstrated an effect for meditation state just missing the $P=0.05$ cut-off $(F(1,15)=4.09, P=0.06)$, with no effect for electrode $(P=0.97)$, and no interaction between electrode and state was observed $(P=0.67)$. Analyses of

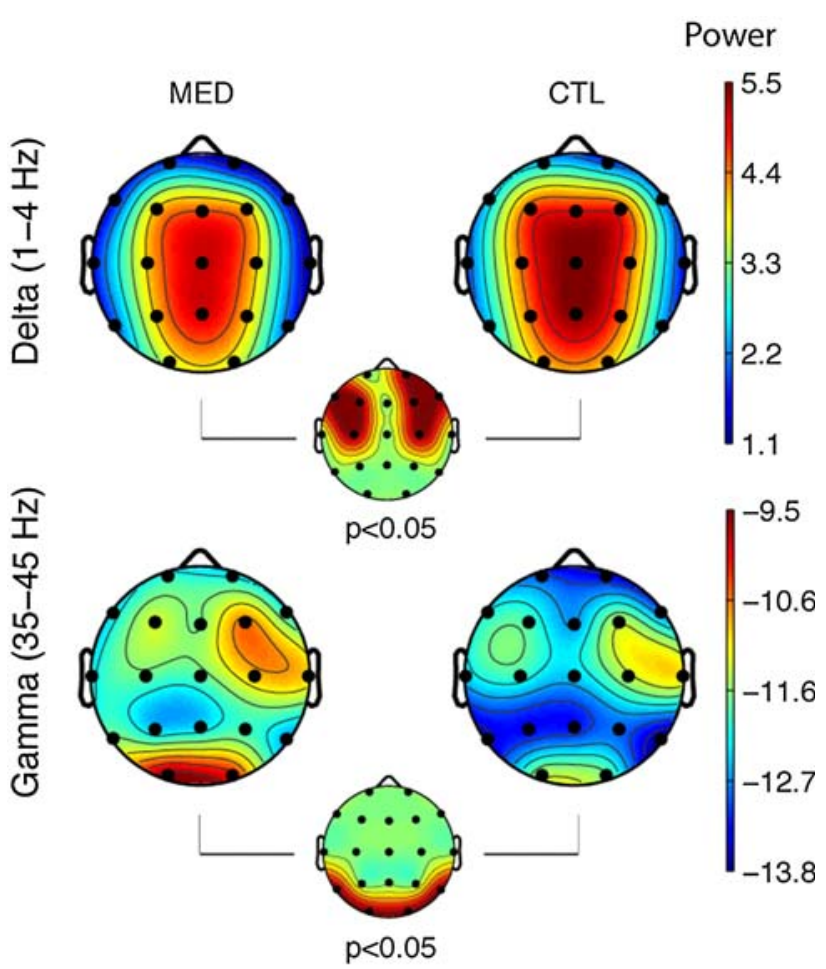

Fig. 2 The scalp maps for delta $(1-4 \mathrm{~Hz})$ and gamma $(35-45 \mathrm{~Hz})$ bands are displayed with meditation on the left and control task on the right. Both bands showed a statistically significant difference comparing meditation and control states and the scalp map indicating statistical significance is shown below. Statistical significance was determined using bootstrap statistics with false detection rate (FDR) correction for multiple comparison with the threshold significance set at $P<0.05$, indicating bilateral frontal decreases in delta power (significant at F3, F4, F7, F8, C3, and C4) and parieto-occipital increases in gamma power (significant at P7, P8, O1, and O2) as meditation state effects. Similar analyses run on the theta, alpha, and beta bands indicated no statistical differences between states at any scalp site and are thus not shown

FP1, F7, FP2, and F8 produced the same pattern of findings.

Theta $(4-8 \mathrm{~Hz})$. Bootstrap analysis with FDR correction for multiple comparisons indicated no main effect of state at any electrodes for theta power during meditation relative to control period. Parametric ANOVA assessment of theta power also yielded no significant effect of state, $(F(1,15)=0.037, \quad P=0.85) \quad$ or electrode location $(F(2,30)=0.96, P=0.39)$. A significant state $\mathrm{x}$ electrode interaction, $F(2,30)=7.75, P=0.002$, indicated with post hoc evaluation that theta power exhibited relative increase during meditation state at $\mathrm{Fz}(P=0.006)$, but not at $\mathrm{Cz}(P=0.99)$ or $\mathrm{Pz}(P=0.85)$.

Alpha $(8-12 \mathrm{~Hz})$. Bootstrap analysis with FDR correction for multiple comparisons indicated no main effect of state at any electrodes for alpha power during meditation relative to control period. Parametric ANOVA assessment of alpha power at midline electrodes likewise produced no 
meditation state effect $(F(1,15)=0.096, \quad P=0.76)$, although a main effect of electrode was found, $F(2,30)=$ $18.8, P=0.000005$, confirming that alpha was the greatest at more posterior sites as expected. No interaction between state and electrode was obtained $(F(2,30)=1.1, P=$ $0.34)$. Post hoc testing confirmed that alpha power was greater at $\mathrm{Pz}$ than $\mathrm{Fz}(P=0.0001)$ and $\mathrm{Cz}(P=0.004)$, and greater at $\mathrm{Cz}$ than $\mathrm{Fz}(P=0.04)$.

A separate ANOVA analysis of alpha power at occipital sites $(\mathrm{O} 1$ and $\mathrm{O} 2)$ was conducted to assess alpha power at the area of maximal power. No state $(F(1,15)=0.055$, $P=0.82)$, electrode $(F(2,30)=1.5, P=0.24)$, or interaction was found $(F(2,30)=0.55, P=0.47)$. Additional separate analyses of low $(8-10 \mathrm{~Hz})$ and high $(10-12 \mathrm{~Hz})$ alpha indicated the same pattern of findings.

Beta $(12-25 \mathrm{~Hz})$. Bootstrap analysis with FDR correction for multiple comparisons indicated no main effect of state for beta power during meditation relative to control period. Analysis of beta power at the midline electrodes indicated no state $(F(1,15)=0.62, P=0.44)$ or interaction $(F(2,30)=$ $1.4, P=0.26)$ effects, but a main effect of electrode was found, $F(2,30)=4.33, P=0.02$, indicating that beta power was greater at more posterior sites. Post hoc assessment found beta power at $\mathrm{Pz}$ was greater than at $\mathrm{Fz}(P=0.03)$, but not significantly different from $\mathrm{Cz}(P=0.92)$; beta power at $\mathrm{Cz}$ was trend-level greater than at $\mathrm{Fz}(P=0.07)$. Additional separate analyses of low (12-15 Hz), medium (15-18), and high $(18-25 \mathrm{~Hz})$ beta indicated the same pattern of findings and are not presented separately.

Gamma $(35-45 \mathrm{~Hz})$. Bootstrap analysis with FDR correction for multiple comparisons indicated a main effect of state with increased gamma power during meditation relative to control period at $\mathrm{P} 7$ and $\mathrm{P} 8$ (both $P<0.05$ ), as well as $\mathrm{O} 1$ and $\mathrm{O} 2$ (both $P<0.01$ ). Parametric ANOVA analysis of the gamma power at midline electrodes demonstrated no state $(F(1,15)=1.26, P=0.28)$, electrode $(F(2,30)=2.22, \quad P=0.14), \quad$ or interaction $(F(2,30)=$ $1.35, P=0.27)$ effects. Additional analysis of gamma power was conducted specifically at occipital electrodes $(\mathrm{O} 1, \mathrm{O} 2)$ because of the greater gamma power found at this location in the bootstrap/FDR analysis. Analysis of occipital gamma power at $\mathrm{O} 1$ and $\mathrm{O} 2$ yielded a significant state effect, $\quad F(1,15)=9.32, \quad P=0.008$, but no electrode $(F(2,30)=2.02, P=0.18)$ or interaction $(F(2,30)=0.59$, $P=0.45)$ effects. Additional analyses including $\mathrm{P} 7$ and $\mathrm{P} 8$ in the ANOVA indicated the same pattern of findings, but as the effect was the greatest at $\mathrm{O} 1$ and $\mathrm{O} 2$, only these data are presented.

Independent component analyses (ICA)

Figures 3, 4, 5 illustrate the grand average scalp maps of the independent components of the three EOG classes or clusters: Fig. 3 illustrates those accounting for vertical and horizontal eye movements. Figure 4 illustrates those associated with the left and right temporalis muscle activity. Figure 5 illustrates the occipital alpha cluster of components. Each subject yielded one to two eye-movement-related components and one to two occipital alpha components for a total of 23 components for both the alpha and eye-movement clusters. Additionally, one to six muscle components were identified per subject for a total of 37 components in the muscle cluster.

Bootstrap statistics with FDR correction across frequencies 1-55 indicated that no difference in spectral frequency power was present between states for either the eye- or the muscle-independent component clusters (see Figs. 3 and 4). A statistically significant increase in gamma ( $>25 \mathrm{~Hz})$ power was found in the occipital alpha independent components (see Fig. 5, gray area at bottom indicates the area of statistical significance comparing between states). Analysis of the specific $35-45 \mathrm{~Hz}$
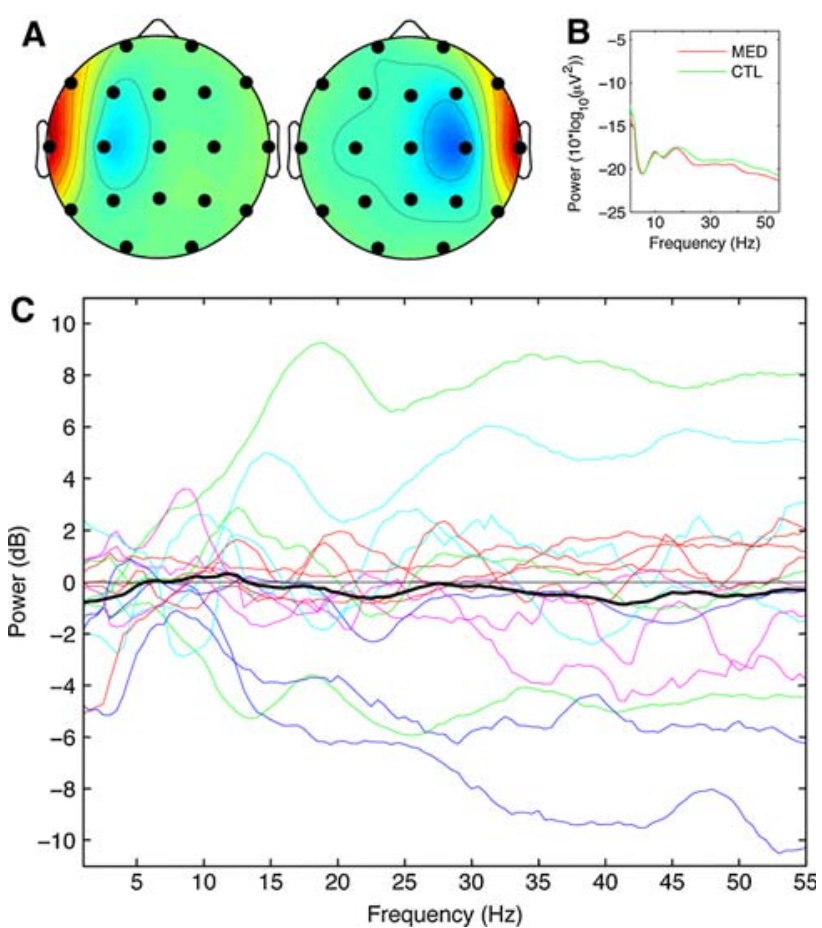

Fig. 3 Panel $A$ indicates the grand average scalp map for the left and right muscle-independent components, respectively. Panel $B$ presents the grand average power spectra for the muscle components for both meditation and control states. In panel $C$, the thin, colored lines indicate difference in power across the range of frequencies for the muscle components; meditation minus control period for all subjects (when more than one component was present for a given subject, the power spectrum for these components was averaged). The bold, black trace indicates the grand average spectrum difference for the contrast meditation minus control such that segments above 0 indicate greater average component activity during meditation and below 0 indicate greater average component activity during control period 
A
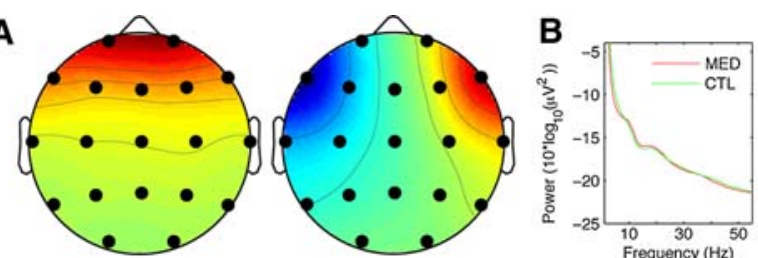

C

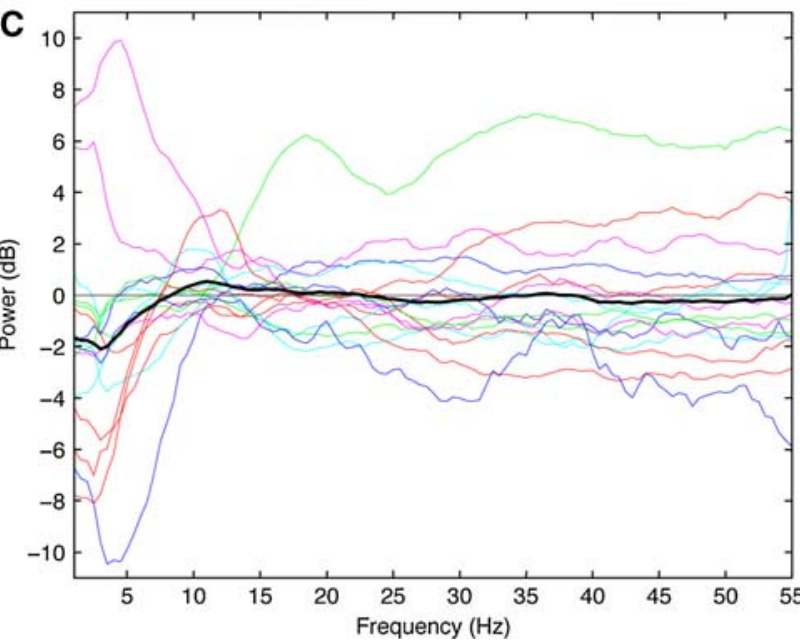

Fig. 4 Panel $A$ indicates the grand average scalp map for vertical and horizontal eye-movement independent components, respectively. Panel $B$ presents the grand average power spectra for the eyemovement components for both meditation and control states. In panel $C$, the thin, colored lines indicate the difference in power across the range of frequencies for the eye-movement components between meditation and control period for all subjects. The bold, black trace indicates the grand average spectrum difference for the contrast meditation minus control such that segments above 0 indicate greater average component activity during meditation and below 0 indicate greater average component activity during control period

frequency gamma band activity for each independent component (IC) class comparing meditation to control state activity indicated that independent components due to muscle ( $t$-test, $d f=15, P=0.55)$ and eye ( $t$-test, $d f=15, P=0.83$ ) demonstrated no difference between meditation and control states. Occipital alpha IC gamma activity was significantly greater in meditation relative to control state using bootstrap statistic with FDR correction for multiple comparisons (bootstrap, $\quad d f=15, \quad P=$ 0.0075 ), but only marginally different between states using parametric statistics ( $t$-test, $d f=15, P=0.07$ ), in any case mirroring the significant difference observed in gamma power at the occipital scalp electrodes. Analysis was applied to the delta power for the eye-movement ICs as this activity reflects typical slow eye movements. Less eye-movement activity was found for the meditation compared to control state, with a marginal parametric difference obtained $(t(1,15)=3.05, P=0.10)$, and a significant non-parametric (Wilcoxon sign test) outcome obtained $(P=0.006)$.
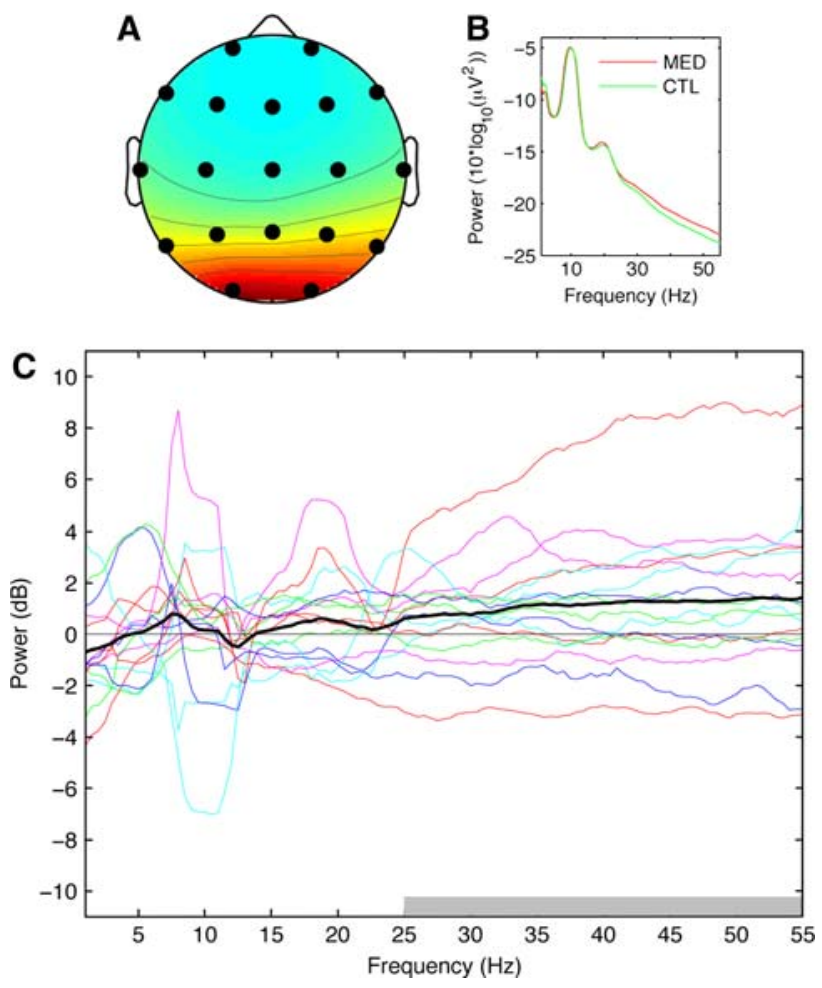

Fig. 5 Panel $A$ indicates the grand average scalp map for the occipital alpha independent components. Panel B presents the grand average power spectra of the occipital alpha components in meditation and control states. In panel $C$, the thin, colored lines indicate the difference in power across the range of frequencies for the occipital alpha components between meditation and control periods for all subjects. The bold, black trace indicates the grand average spectrum difference for the contrast meditation minus control. The gray bar at the bottom of the figure indicates frequencies over which bootstrap statistics with FDR correction for multiple comparisons indicated statistically significant greater values in meditation than control state $(25-55 \mathrm{~Hz})$

\section{Additional analyses}

Correlations were calculated to explore the relationships between both the eye-movement-related IC delta power (eye) and scalp-recorded delta power as well as between gamma power (muscle, eye) and scalp-recorded gamma power, so as to assess possible non-cortical sources for the observed state effects of meditation. Specifically, correlations were computed between the gamma power in the eye- and muscle-independent components and the scalprecorded gamma power across midline as well as occipital electrodes in each experimental state. To assess the possibility that slow eye-movement differences between meditation and control state might contribute to the occipital gamma findings, correlations between eyemovement independent component delta power and gamma power at scalp channels were also computed. As the increased delta activity observed at fronto-lateral locations might be related to eye-movement activity not 
fully removed from the data, additional correlations were run between delta power in the eye-movement independent components and delta power in the (post eye-movement independent component removal) midline and frontal channel data.

There were no correlations between delta activity in the eye-movement IC's and midline or frontolateral electrodes in either meditation or control states: meditation- $\mathrm{Fz}, r=$ $0.044, P=0.87, \mathrm{Cz}, r=0.00, P=1.00, \mathrm{Pz}, r=0.16$, $P=0.56, \quad$ F3 $, \quad r=0.22, \quad P=0.42, \quad$ F4,$\quad r=0.084$, $P=0.76 ;$ control-Fz, $r=-0.17, P=0.52, \mathrm{Cz}, r=$ $-0.087, P=0.75$, at $\mathrm{Pz}, r=-0.051, P=0.85, \mathrm{~F} 3$, $r=0.094, P=0.73, \mathrm{~F} 4, r=0.016, P=0.95$.

In contrast, significant correlations between gamma power in the muscle IC cluster and gamma power at midline electrodes were obtained for both state conditions: meditation-Fz, $r=0.61, P=0.013, \mathrm{Cz}, r=0.60, P=$ $0.014, \mathrm{Pz}, r=0.47, P=0.066$; control-Fz, $r=0.55$, $P=0.028$, at $\mathrm{Cz}, r=0.46, P=0.073$, at $\mathrm{Pz}, r=0.32$, $P=0.22$. Significant correlations between gamma power in the eye-movement IC cluster and gamma power at midline electrodes were also obtained for the control condition with trends observed also at $\mathrm{Fz}$ in meditation: meditation-Fz, $r=0.50, P=0.058, \mathrm{Cz}, r=0.41, P=$ $0.13, \mathrm{Pz}, r=0.081, P=0.78$; control-Fz, $r=0.82$, $P=0.0001, \quad \mathrm{Cz}, \quad r=0.63, \quad P=0.012, \quad \mathrm{Pz}, \quad r=0.54$, $P=0.039$. Importantly, no correlations were found between gamma power at occipital electrodes and gamma power in the muscle IC cluster (meditation-O1, $r=0.11, P=0.69, \mathrm{O} 2, r=0.17, P=0.53$; control-O 1 , $r=-0.061, P=0.82, \mathrm{O} 2, r=-0.093, P=0.73)$ or the eye-movement IC cluster (meditation-O1, $r=-0.04$, $P=0.89, \quad$ O2 $, \quad r=0.014, \quad P=0.96 ; \quad$ control-O1, $r=0.38, P=0.17, \mathrm{O} 2, r=0.19, P=0.49$ ). The correlations between the occipital alpha IC gamma activity and scalp-recorded gamma power were of moderate significance at midline electrodes: meditation-Fz, $r=0.42$, $P=0.104 ; \quad \mathrm{Cz}, \quad r=0.38, \quad P=0.15 ; \quad \mathrm{Pz}, \quad r=0.47$, $P=0.064 ; \quad$ control-Fz, $\quad r=0.50, \quad P=0.048 ; \quad \mathrm{Cz}$, $r=0.60, P=0.013 ; \mathrm{Pz}, r=0.70, P=0.003$. These correlations were, however, quite significant at occipital electrodes: (meditation-O1, $r=0.77, P=0.0001, \mathrm{O} 2$, $r=0.71, P=0.002$; control $-\mathrm{O} 1, r=0.79, P=0.0001$, $\mathrm{O} 2, r=0.75, P=0.001$.

\section{Covariate analyses}

Additional analyses were conducted using covariates to characterize individual differences underlying the spectral power findings. The primary covariates were those relating to order of experimental conditions, reported drowsiness during the experimental sessions, and intensity of meditation practice (number of years of daily meditation practice, current number of hours/day of meditation practice). Significant interactions for some of these covariates were obtained for the delta, alpha, and gamma bands, with no reliable effects obtained for theta or beta power.

\section{Delta}

A significant interaction among state, order of experimental sessions (meditation $\rightarrow$ control vs. control $\rightarrow$ meditation), and midline electrode location was found, $F(2,28)=4.70, \quad P=0.017)$. This outcome suggests decreased midline delta power during meditation relative to rest specifically for those participants doing the control period prior to the meditation period, but not those meditating first. Breakdown of this interaction with Tukey post hoc testing indicated that when the control period occurred first, midline delta power was decreased in the subsequent meditation session at $\mathrm{Fz}(P=0.0004)$ and $\mathrm{Cz}$ $(P=0.027)$ but not $\mathrm{Pz}(P=0.30)$. No differences among midline electrodes for delta power were found in participants meditating first $(\mathrm{Fz}, P=1.0 ; \mathrm{Cz}, P=0.77 ; \mathrm{Pz}$ $P=0.15)$. A second covariate interaction was found for the state $\times$ reported drowsiness $\times$ midline electrode location during meditation, $F(2,28)=3.39, P=0.06$ ), indicating that only those subjects not reporting drowsiness during meditation showed a tendency for decreased midline delta power in meditation. No significant outcomes were found for state effect at lateral-frontal locations.

\section{Alpha}

The interaction among order of experimental session $\mathrm{x}$ state effects $\mathrm{x}$ midline electrode location was significant, $F(1,14)=5.31, P=0.037$. For the occipital electrodes the order $\times$ state interaction was reliable, $F(1,14)=4.00$, $P=0.065$, with both such interactions indicative that whichever experimental period occurred second in the experimental order scheme tended to produce more alpha power (See Fig. 6a). The interaction among state x meditation daily practice was trend-level significant for the midline, $F(1,14)=3.89, P=0.068$, as well as occipital electrodes, $F(1,14)=3.99, P=0.066$. These outcomes suggested that more years of meditative practice tended to correlate with slight decreases in alpha power during meditation, whereas fewer meditation practice years correlated with trend increases in alpha power during meditation (See Fig. 6b). The state $\times$ drowsiness $\times$ electrode interaction was marginally significant, $F(2,28)=3.06$, $P=0.071$, such that participants reporting drowsiness during the control period tended to produce decreased alpha in the control relative to meditation period, with the opposite pattern obtained for those not reporting such 
drowsiness. The same but weaker interaction was observed for occipital alpha power $(F(2,28)=2.44, P=0.14)$.

\section{Gamma}

A number of covariates produced significant findings for both the midline and occipital gamma power (reported drowsiness, number of hours of current daily meditation practice). Given the fact that the muscle IC cluster gamma power correlated with the gamma power at midline electrodes and that the same pattern of significant findings obtained with midline gamma covariate analysis obtained with muscle IC gamma covariate analysis, these associations
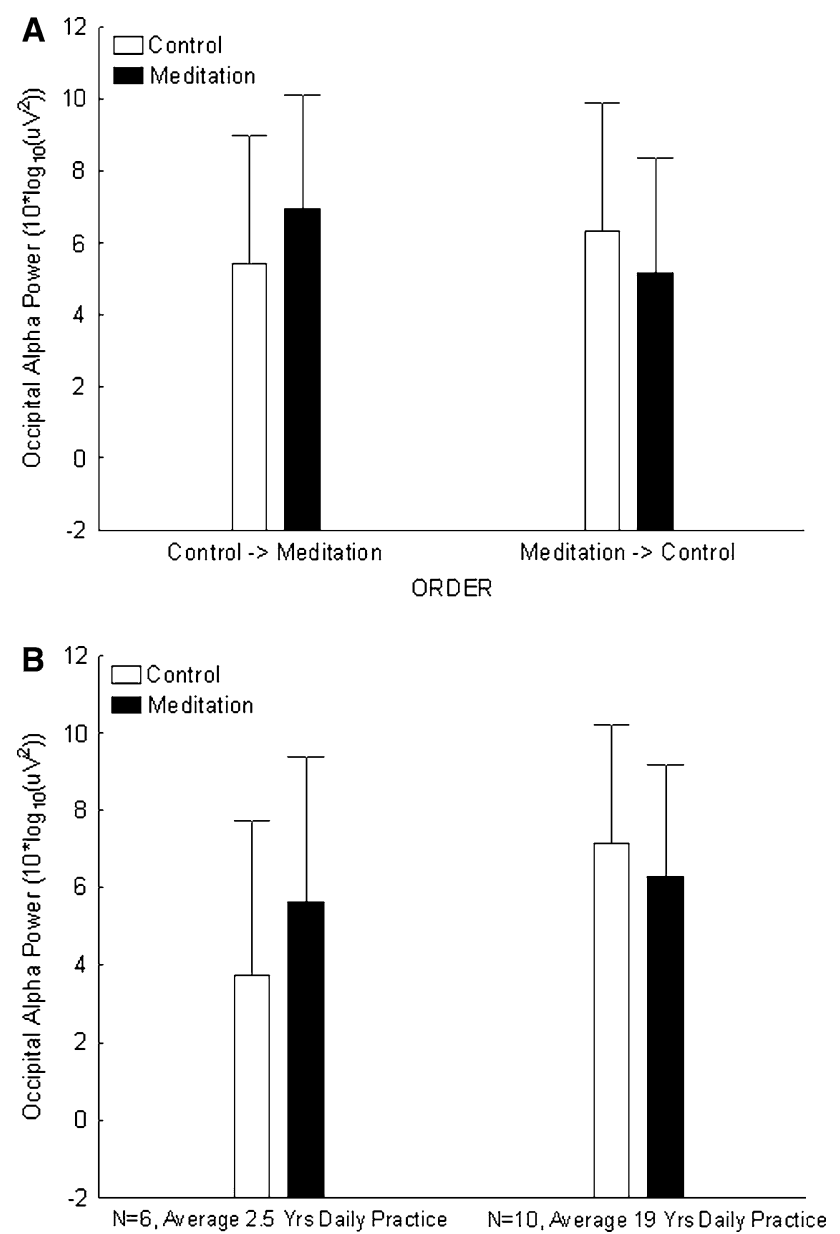

Fig. 6 a Experimental order and alpha power. Participants undergoing the control period followed by the meditation period tended to evince greater alpha power during meditation, whereas those undergoing the meditation period followed by the control period tended to evince greater alpha power during the control period. b Meditators with less than 5 years of daily meditation practice ( $n=6, \mathrm{M}=2.5, \mathrm{SD}=1.4)$ vs. meditators with 10 and greater years of daily meditation $(n=10, M=19.3, \mathrm{SD}=8.5)$ practice showed different tendencies with regard to alpha power across experimental sessions, with shorter-term practitioners showing a trend toward greater alpha during meditation and longer-term practitioners showing the opposite pattern are likely due to muscle rather than brain reactivity and are not reported.

No reliable outcomes were obtained when the self-report score for meditative depth between meditation and control sessions was used as a covariate for gamma power. Categorization of participants into those with a history of $10+$ years $(n=10, M=19.3, \mathrm{SD}=8.6$ years) and those with $<10$ years ( $n=6, M=2.5, \mathrm{SD}=1.4$ years) of daily meditation practice yielded significant interactions for occipital gamma. The long-term meditator category yielded a significant main effect, $F(1,14)=4.87, P=0.044$, indicating that the sub-group reporting more years of daily meditation practice exhibited greater gamma power across task conditions (See Fig. 7a, b). In addition, an interaction among state $\times$ daily meditational practice length $\times$ electrode was found, $F(1,14)=4.53, P=0.05$. Specifically, gamma power was increased in meditation vs. control state in long-term meditators $(\mathrm{O} 1, P=0.0002$; $\mathrm{O} 2, P=0.008$ ) but only marginally in relatively short-term meditators (O1, $P=0.76$; O2, $P=0.09$ ). The level of drowsiness $\times$ state interaction was also reliable, $F(1,14)=5.41, P=0.035$. Gamma power increases were significant only for those participants not reporting drowsiness during rest (Tukey post hoc comparison $P=0.0069$ ) and were absent for the group who did report drowsiness (Tukey post hoc comparison $P=0.57$ ). In contrast, the self-reported drowsiness during meditation state did not show a significant interaction with gamma.

\section{Discussion}

Meditation and neuroelectric measures

Vipassana meditative practice involves the adoption of a mindful and receptive mental awareness, with attentional absorption on present-moment sensations in the body and meta-cognitive reframing of ongoing experience as impersonal phenomena to be observed but not reacted to (Gunaratana 2002; Hart 1987; Lutz et al. 2007). EEG measures were obtained from experienced Vipassana meditators with conditions that contrasted the meditative state with a control cognitive condition designed to mimic "everyday thinking." The pattern of meditation-induced increase in parieto-occipital gamma activity, concomitant decrease in frontal delta power, and a shift to a more frontal distribution of theta activity suggests that sensory processing and cognitive processing were altered during meditation relative to the control state. However, the typically reported meditation state changes in the theta and alpha frequencies were not found (Cahn and Polich 2006).

Delta $(1-4 \mathrm{~Hz})$ power is known to correspond with inhibitory functions (Babiloni et al. 2006; de Jongh et al. 

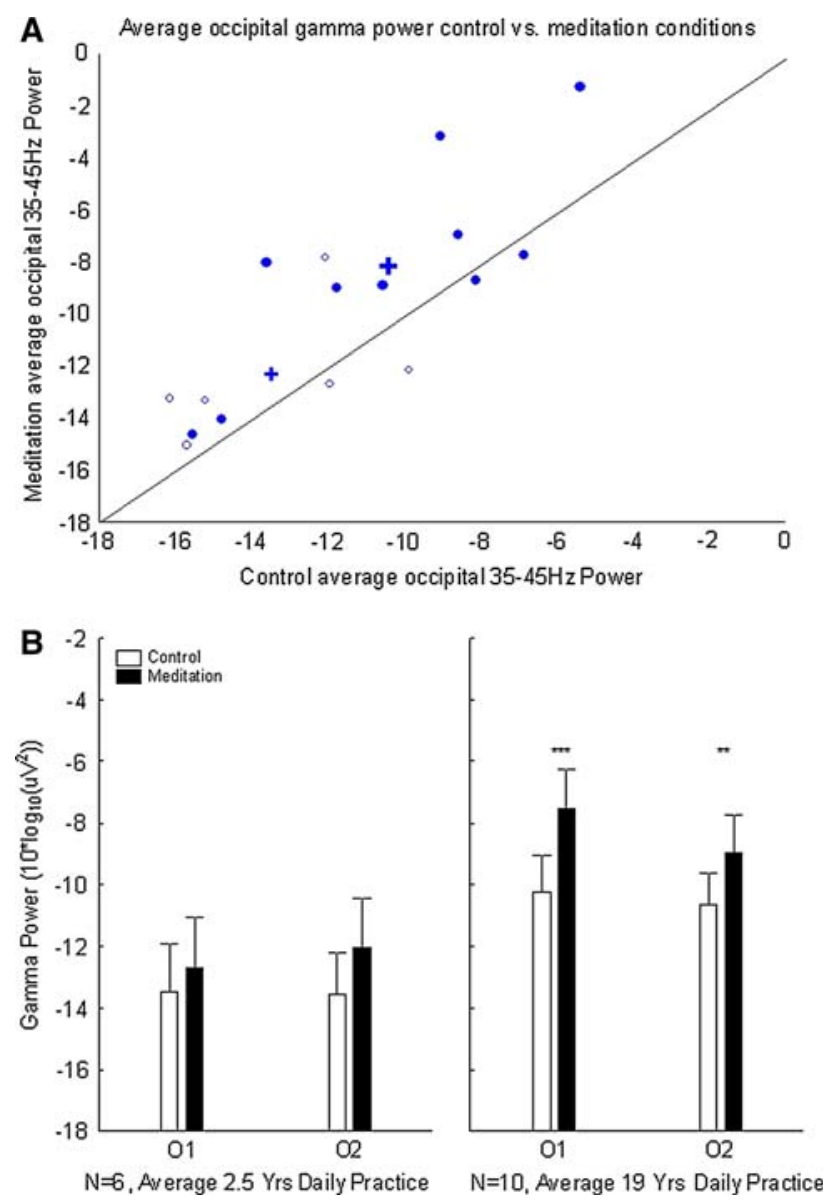

Fig. 7 a Meditators with 10 and greater years of daily meditation practice in solid circles $(n=10, \mathrm{M}=19.3, \mathrm{SD}=8.5)$, Meditators with less than 5 years of daily meditation practice in solid circles $(n=6, \mathrm{M}=2.5, \mathrm{SD}=1.4)$, smaller " + " indicates the mean average occipital gamma in control vs. meditation conditions in shorter-term meditators, larger "+" indicates the mean average occipital gamma in control vs. meditation conditions in longer-term meditators. b Interaction between meditation expertise and occipital gamma effect

2001; Niedermeyer and Lopes da Silva 1999; Penolazzi et al. 2008) and has been reported to be associated with meditation only recently, with a trait increase in frontal delta power reported for both Zen and Qi-Gong practitioners (Faber et al. 2008; Tei et al. 2009). Theta (4-8 Hz) power is known to correspond with meditation, although the cortical sources for these effects are still not fully understood. Frontal mid-line theta tends to be associated with concentrative attentional engagement, whereas less specific topographic theta distribution increases are observed in periods of drowsiness (Basar et al. 2001a, b; Mitchell et al. 2008). Alpha (8-12 Hz) power corresponds with cortical idling, cortical suppression, and relative deactivation of underlying brain areas (Niedermeyer 1997). Gamma $(35-45 \mathrm{~Hz})$ power is known to correspond with stimulus representation and feature binding, possibly coupling tightly with perceptual awareness (Fries et al. 2001, 2008) and selective attention (Fell et al. 2003). The relative impact of meditation on different frequencies of brain activity are still not well understood across meditative practices and is likely both practice-specific and related to differential effects early vs. late in the learning process.

Important to the interpretation of the present relative to past findings is that previous assessments often have not obtained neuroelectric measures during meditation vs. cognitive control periods of equal length. Furthermore, a "resting" state is not likely the same for meditators compared to non-meditators, as meditators often report an inability for non-meditative resting. Indeed, in the present study, a number of participants reported difficulty in avoiding engagement in meditative practice with eyesclosed and the posture used during meditation even with the instructions to keep the mind engaged with neutral memories during the control period. Nonetheless, although a few participants reported approximately the same meditation depth in both periods, the consistent rating of a greater meditative depth in the meditation period than the control period likely reflects a different subjective experience of the two states. Further, both the neuroelectric measures and the introspective meditative depth differentiated between meditation and the control condition.

\section{Delta effects}

Previous assessments of meditation have not often reported effects on the delta frequency band, but it is unclear whether it has been systematically analyzed. Recently, two reports of increased trait frontal delta in long-term Zen and Qi-Gong meditators suggest that there may be an important interaction between meditative practice and delta brain activity (Faber et al. 2008; Tei et al. 2009). Increased frontal delta in long-term meditators may indicate a functional inhibition of brain appraisal systems in line with a detachment from analysis, judgment, and expectation (Tei et al. 2009). In this study, decreased delta activity in the temporo-parietal junction, secondary motor cortex, and sensory association cortices appeared indicative of increased brain activation associated with detection and integration of internal and external sensory information, with detachment from the same as indexed by inhibited activity (increased delta) in prefrontal areas responsible for analyzing, judging, and expectation (Tei et al. 2009).

The present study found a significant state effect in the delta frequency band, such that the meditation state was characterized by a decrease in bilateral frontal delta power, indicative of an increase in frontal activation during Vipassana meditation relative to the control condition. It is worth noting that this frontal delta decrease was significant 
in the bootstrap with FDR correction for multiple comparison statistical analysis of the scalp channel data at the frontal electrodes, but just missed statistical significance by standard parametric ANOVA testing $(P=0.06)$. It has been argued that bootstrap statistics may be of greater theoretical utility in application to relatively non-Gaussian measures such as EEG spectral power values (Darvas et al. 2004; Delorme 2006), suggesting that the significant finding here with bootstrap statistics is quite valid, but it should be noted that the parametric testing result was trendlevel. It is important to note that the delta power in the eye-movement-related independent components during meditation relative to control states was also decreased reflecting less eye movement during meditation. However, the eye-movement-related delta power did not correspond with the scalp-recorded delta power, indicating that the eye-movement artifact rejection was effective and that the decrease in delta power measured at the frontal scalp electrodes was separate from meditation's inhibitory effect on eye movements. One significant covariate in the analysis of midline delta activity was self-reported drowsiness during meditation, and this covariate showed a trend significance $(P=0.09)$ when used in the ANOVA analysis of lateral frontal delta as well. Those participants reporting drowsiness during meditation did not appreciably contribute to the decrease in frontal delta power seen during meditation, further suggesting that this delta power decrease is a marker of the more highly aware state seen in meditation relative to control state.

It is possible that the delta power decrease we report here as a state effect may be correlated with the increase in baseline delta as a trait effect in previous reports, consistent with the notion that through sustained engagement of frontal circuits during meditative practice, practitioners may train other baseline frontal circuits associated with elaborative processing such as judging, analyzing, and expecting downward. These hypotheses are suggestive but provide important footholds for theoretical development of the relationship between meditation and EEG measures.

\section{Theta effects}

No absolute difference in theta power between the meditation and control states was observed. A significant interaction between state and electrode location was found that suggested a more frontal distribution of theta activity was present during the meditation state. The implications of this outcome are uncertain but likely reflect the operation of enhanced attentional mechanisms mediated through anterior cingulate engagement (Cahn and Polich 2006). It is of note that a number of recent studies have found strong increases in frontal theta power during concentrative/focused attention meditation states (Aftanas and Golocheikine 2001,
2003; Baijal and Srinivasan 2009) and that frontal theta power is thus a key contributor to meditative neural dynamics that likely shows differential engagement dependent on the specific technique employed.

\section{Alpha effects}

Meditation and alpha power effects in the long-term Vipassana practitioners were absent comparing meditation and control states, which supports the assertion that alpha increases often reported in early studies of meditation were related to assessing beginning meditators vs. experts. While meditation state did not affect alpha power the order with which participants engaged in the meditation vs. control tasks was significant-i.e., whichever task was engaged later tended to have greater associated alpha power (see Fig. 6a). This interaction between order and alpha power was significant at midline electrodes and trend-level at occipital electrodes. A number of early studies on meditation utilizing a fixed-order design for the engagement in control task (often the non-specific instruction to "rest") followed by meditation may have shown increases in alpha activity actually related to the passage of time within the study rather than anything specific regarding meditation. Nonetheless, given the large number of studies reporting alpha state and trait effects in the EEG literature, it is also possible that some forms of meditative practice may be more reliably associated with alpha state effects, and/or that there are alpha state effects toward the beginning of regular practice that dissipate with the development of expertise.

Supporting this latter interpretation, occipital alpha power in this study was somewhat related to meditation expertise, as participants with $10+$ years daily practice ( $n=10, M=19.3$ ) tended to demonstrate more similar alpha power levels across states, whereas those subjects meditating $<10$ years of daily practice $(n=6, M=2.5)$ tended to demonstrate enhanced alpha power during meditation (see Fig. 6b). Longitudinal studies assessing the impact of meditation over time in large samples are necessary to substantiate the hypothesis of increased state and trait alpha power resulting from meditative practices at different points in the learning process. The current findings support that meditative practice may enhance alpha power in the beginning stages of learning and that with expertise a trait-level increased alpha power may develop after which meditation state effect is no longer characterized by alpha enhancement (Cahn and Polich 2006). Direct support for this hypothesis also would require demonstrating higher trait alpha in the longer-term meditator participants, which was not observed in the present sample. An association between intensity of meditative practice and alpha power was obtained; however, as participants with 
$+2 \mathrm{~h}$ daily practice demonstrated higher baseline alpha across states for the occipital alpha independent components. Further studies with greater numbers and multiple meditator cohort groups (multiple techniques and experience levels) are clearly needed to substantiate the alpha trait and state hypotheses.

\section{Gamma effects}

Gamma rhythms $(30 \mathrm{~Hz}$ and above, often reported as centered around $40 \mathrm{~Hz}$ (Basar-Eroglu et al. 1996)) have been widely characterized as significantly related to momentary contents of consciousness (Sauve 1999). Further, electrical activity in this high frequency range has been shown to be a possible candidate for the neurophysiologic substrate of the "binding" of multiple aspects of conscious experience and perceptions into the coherent subjective state of moment-to-moment awareness (Singer 1993; Varela 1995). Evidence has accumulated supporting the notion that enhanced gamma synchrony and/or power in appropriate cortical areas is critically associated with both perceptual events (Gross et al. 2007; Lachaux et al. 2005; Meador et al. 2002; Rodriguez et al. 1999) and readiness to perceive periliminal and/or ambiguous stimuli (Melloni et al. 2007). While we hypothesized finding a meditation-related gamma power increase in frontal and/or parietal areas, reflecting increased functional processing in frontal and/or somatosensory cortices related to body sensation and/or executive control, instead we found increases in occipital areas. The increase in occipital gamma synchronization found in our current sample may indicate that this open-awareness meditative state involves a more sensitive and perceptually clear awareness of momentto-moment experience. The reasons for an occipital distribution are certainly not clear but may be specific to the Vipassana meditative technique as previous reports have not found such gamma increases associated with other meditative techniques. Further, the fact that both baseline and meditation-related increases in occipital gamma power and were found to significantly covary with meditational expertise as indexed by total years of daily practice (see Fig. 7) suggests that there may be both a state and trait effect of increased gamma power associated with Vipassana practice. Clearly additional studies employing a nonmeditator control group are needed to further substantiate this possible trait effect finding.

Of possible relevance to the parieto-occipital increase in gamma power with respect to Vipassana meditation state, a recent report indicated increased gamma power in this approximate distribution during imagined actions (de Lange et al. 2008). Although Vipassana meditation practice does not invoke imagined actions, it does involve the repetitive scanning of body sensations from head to toes, which may recruit some surreptitious access to either imagined body action and/or visualization of body parts as the scanning occurs. Participants were not specifically asked to report on their visual experiences during the meditation and control sessions, but no one indicated a strong visual component on their free-form written summaries of the meditation state. This distribution of gamma power is therefore not readily explained via known properties of Vipassana practice, and it may be related to properties of widespread posterior gamma increases not currently understood. Finally, it is also noteworthy that these increases did not correlate with reported depth of meditation but did correlate with increased number of years of daily meditation practice and likely meditative expertise.

Early gamma reports in meditation. Previous findings of widespread gamma increases in meditation are mostly limited to early studies prior to the development of sophisticated computerized methods for the quantitative EEG analysis and the separation of artifact from cortical signals (Anand et al. 1961b; Banquet 1973; Das and Gastaut 1957), although a few more recent meditation gamma findings have been reported as well (Aftanas and Golosheykin 2005; Lehmann et al. 2001; Lutz et al. 2004). Das and Gastaut first reported widespread increased high frequency $(20-40 \mathrm{~Hz})$ activity in association with meditation, reporting that after a long period of meditation some of the more advanced Yogis studied exhibited increased gamma states associated with periods of subjective deep meditation/samadhi (Das and Gastaut 1957). Anand et al. (Anand et al. 1961b) reported that "fast waves" were observed in the EEG recordings from a Yogi meditating in a box for a period of 2-3 days, but a separate comprehensive report on the EEG records from this case study participant and others with similar expertise did not mention this finding, instead noting the pronounced lack of alpha blocking exhibited while these participants were in meditation (Anand et al. 1961a). Banquet reported increased 20 and $40 \mathrm{~Hz}$ activity in a subset of TM practitioners who reported experiencing a deep meditative/transcendent subjective state during the EEG recording (Banquet 1973), replicating Das and Gastaut's assertion that deep transcendent states of meditative consciousness may be marked by increases in gamma activity.

Recent reports of gamma and meditation. In consideration of recent findings relating gamma to conscious experience and the early suggestive gamma findings summarized earlier, Ott (2001) specifically hypothesized gamma activations in deep meditation, possibly correlated with intensive wakefulness and all-encompassing unity. No such meditation state effects of gamma power were found, however, and instead the increases in gamma observed in some subjects were only those associated with movement artifacts. However, this study used only one electrode $(\mathrm{Cz})$ 
so that this study can not be taken as a comprehensive gamma assessment. A case study of an advanced Tibetan Buddhist meditation teacher/practitioner indicated that gamma $(35-44 \mathrm{~Hz})$ was the most reliable frequency band distinguishing between different meditative states in this single individual (Lehmann et al. 2001). They specifically reported that relative occipital increases in gamma were observed in a meditation focused on visualization of the Buddha relative to other meditative states not incorporating visualization. Another recent study of meditation and gamma activity indicated that long-term meditators relative to controls exhibited decreased negative emotional stimulus-induced gamma power activation, likely related to decreased emotional reactivity due to such practice (Aftanas and Golosheykin 2005).

The one recent previous report of widespread increases in gamma power assessed expert Tibetan Buddhist meditators engaged in a loving-kindness/compassion meditative practice and found both trait and state associations between meditation and gamma activity (Lutz et al. 2004). Midline frontoparietal gamma power was higher at baseline (trait) in advanced Tibetan Buddhist practitioners, and upon engaging in compassion meditation gamma power increased in magnitude (state) to a very significant degree. The topography of the meditation state effect was located bilaterally over the parieto-temporal and mid-frontal electrodes. The outcomes suggested that increased gamma may be related to a change in the quality of moment-to-moment awareness, as claimed by the meditation practitioners. Further, a reliable association between the estimated total hours in meditative practice and the baseline gamma power was found that implied attention and affective processes are flexible skills which can be trained and that gamma activation may be a marker for these processes.

The gamma state effect of meditation in the present study is similar but refers to a different meditative technique with a different topography obtained, although it is of note that we employed a mastoid reference, whereas the previous study employed average reference. A striking similarity in the pattern of results obtained across the studies is seen in the fact that the previous study found higher baseline gamma power in the expert meditator group than the non-meditator group as well as meditationinduced increases in gamma power; similarly, the meditation expertise covariate analysis of the present gamma findings indicated greater baseline occipital gamma power as well as meditation state-induced increases in this activity for the more experienced participants. The previous study employed Tibetan Buddhist/Mahayana meditators practicing a non-referential compassion technique, whereas long-term practitioners of Vipassana as taught within the Theravadan/Hinayana tradition were assessed in the present study. The presently-reported Vipassana technique involves attentional absorption in moment-to-moment subtle sensations of the body concomitant with an attentional stance of non-reactive mindful awareness/open monitoring.

A significant commonality across the states assessed by these two studies is the specific inclusion of a mindful/ open-monitoring component to the practice. Lutz et al. (2004) asserted that the assessed state was an objectless state of mind, involving a dissipation of the object-oriented aspect of experience. Vipassana practitioners report that they are able to adopt a wide-open awareness during practice that is characterized by a subtle yet rich somatosensory experience (Gunaratana 2002), but whether that experience serves as an "object" of attention is likely experienced differently across such meditators. It may be that the widening of the attentional spotlight involved in these meditational practices correlates with the finding of a gamma state effect rather than an effect on the slower theta or alpha frequencies more commonly reported in the past.

\section{Gamma effects and artifact}

It is important to note the well-known artifact from muscles of the scalp, head, and neck that can generate high frequency electrical in the gamma range and to address the possibility that the gamma increases we recorded at the scalp may be muscle related. In addition, significant attention has now been drawn to the fact that microsaccades are significantly associated with increased gamma power (Yuval-Greenberg and Deouell 2009). The multiple analyses conducted utilizing ICA methods to isolate non-brain-related activities such as eye and muscle artifact from the scalp-recorded data were performed to counter this possibility (Jung et al. 2000a). Given that gamma activity artifact from eye movements have been correlated with saccades (Reva and Aftanas 2004; Yuval-Greenberg and Deouell 2009) and microsaccades (Dimigen et al. 2009; Yuval-Greenberg et al. 2008) and that the present subjects were recorded in an eyeclosed state, the probability of eye movements contributing to the gamma finding seems unlikely.

Nonetheless, this possibility was quantitatively assessed by analyzing the activity of the artifactual independent component clusters that account for the eye-movement activities across the two states. Figure 4 illustrates the results, which indicate that there is no difference in the activities of these independent component clusters between states at frequencies above $\sim 6 \mathrm{~Hz}$ including the gamma band. At frequencies below $6 \mathrm{~Hz}$, a small decrease in eye movements in the meditation state relative to control was observed. This outcome implies a tendency toward a decrease in the slow eye movements often observed in eyes-closed conditions during meditation relative to the control task. In sum, eye movements are a very unlikely 
cause for the measured increased gamma power during meditation.

With respect to the more plausible possibility that scalp muscle activity contributed to the increase in parietooccipital gamma observed, we note first that visual inspection for artifact tended to show decreased muscleand movement-related artifact in meditation relative to control task. As is routine, increases in phasic muscle tension and movement were observed in a small number of epochs during both states, but more total epochs were removed from the control state data than from the meditation state data at the level of visual epoch-rejection preprocessing (remaining epochs analyzed out of 180 were $170.4 \pm 13.8$ for the meditation state and $164.1 \pm 26.6$ for the control state). Muscle-related independent components were empirically assessed as an objective check of the possible contribution of muscle activity to the observed effects. Independent components that resembled the wellknown characteristics of muscle activity in terms of focal scalp distribution (often centered at temporal electrodes overlying the temporalis muscle) and featuring relatively high-amplitude high frequency signals were assessed (Fig. 3). No difference in the gamma (or any other frequency) activity of the muscle components was found between meditation and control state. Correlation analyses also were performed on the gamma activity in the muscleindependent components in meditation and rest vs. the scalp-recorded gamma power data. These analyses indicated significant correlation between the rest and meditation muscle IC gamma activations and the gamma activity recorded at midline electrodes, but no correlation with the gamma activity recorded at parieto-occipital electrodes where the significant increase gamma power during meditation was observed.

A last line of evidence further bolstering the claim that the observed gamma effect is cortical in origin is that the occipital alpha independent components also exhibited a gamma state effect. The increase in gamma power of the occipital alpha reflected by the independent components during meditation relative to control state suggest that significant gamma activity may be related to the common cortical source shared by each band. The likelihood of muscle-related gamma co-localizing with cortical-generated alpha activity after ICA decomposition is not significant given that the algorithm is a category of blind source separation known to segregate time series signals according to different spatial and causal generators (Comon 1994; Delorme et al. 2007; Hyvärinen and Oja 2000). In summary, we found that the gamma power increases during meditation in our meditator participants were not correlated with increases in scalp muscle or eye-movement-related activity as assessed by independent component analysis and that instead the occipital alpha independent components demonstrated a meditation state effect in the gamma band. Our report is the first to our knowledge that uses these advanced signal-processing techniques to clearly demonstrate that the occipital gamma during meditation effect we observed is not artifactual.

\section{Choice of control task}

It is possible that the sort of "instructed" mind-wandering state experienced by the present meditators was not consistent with the off-task mind-wandering assayed in the literature on mind-wandering to date (Smallwood et al. 2008, 2007; Smallwood and Schooler 2006). Continued assessment of the state effects of meditation require careful consideration of the control task to employ as well as the psychometric tests to use to assess the actual experiences encountered in the control vs. meditation task. With respect to the various "control tasks" that are used to assess meditation effects, the present introspective data regarding drowsiness in relation to meditative expertise may be instructive. Participants with more years of daily meditation practices reported less drowsiness specifically during the control cognitive condition, and not the meditation condition, whereas participants with greater current number of hours of daily practice were specifically less likely to report drowsiness during meditation but not necessarily control conditions. This outcome may imply that the longterm practice of meditation increases the tendency to maintain alertness even during boring tasks (e.g., instructed mind-wandering), whereas the intensity of current practice is more strongly associated with maintaining alertness during meditation itself. Whatever the choice of control task, one of the current challenges in meditation research is to more fully explore the psychometric characterization of the control state, whether that be the no-instructions "resting state" often assessed or a more controlled cognitive engagement state such as mental calculations or the instructed mind-wandering assayed here. This is of special relevance also to the notion that meditative practices change the ongoing experience of the world in a way that may significantly affect the neural "default network" activity mirroring the reported decreases in elaborative and ruminative processing resulting from such practices (Pagnoni et al. 2008; Tei et al. 2009).

One limitation of the present study is the lack of a control group of non-meditators, such that possible EEG trait measures were not directly assessed, although the significant covariate indicating that greater length of daily meditation practice was associated with the increased gamma meditation state effect is suggestive. Although it is possible that non-meditators might have shown a similar "meditation" state effect reflected by some aspects of demand characteristics for the two cognitive tasks assayed, 
the significant covariate relating meditational expertise to increased gamma makes this possibility less likely. The motivation for staying alert may have varied across the two experimental periods due to some sort of "performance" pressure in the meditation period, thereby leading to higher levels of arousal and possibly confounding the results. This outcome also is unlikely given that analysis of the spontaneous EEG data indicated no changes in power for theta and alpha frequencies between the two states, and increased arousal is generally associated with higher P3 amplitudes, whereas the meditation effects demonstrated here include decreased P3a amplitude (Cahn and Polich 2009; Polich 2007; Polich and Kok 1995).

\section{Theoretical perspectives}

Previous findings with this meditator cohort demonstrated decreased engagement of the frontal attentional systems of the brain to auditory distracters during meditation relative to the control period as indexed by decreased frontal N1 and P3a event-related potential (ERP) component amplitude to distracter and decreased P2 amplitudes to oddball stimuli. These findings occurred concomitantly with a marginal increase in N1 amplitudes to the standards and oddballs, implying that the Vipassana meditation state is associated with intact/enhanced sensory processing together with top-down control of elaborative attentional engagement with the contents of awareness (Cahn and Polich 2009). This outcome is consistent with previous reports of early studies suggesting that meditation may produce a state of brain processing less susceptible to stimulus-driven processing as indexed by alpha blocking (Anand et al. 1961a; Kinoshita 1975; Lehrer et al. 1980). It is also consonant with a number of recent reports noting the enhancement of neural signatures of attentional stability and efficiency due to meditation interventions (Lutz et al. 2009; Slagter et al. 2007, 2009).

The present finding of increased parieto-occipital gamma activity is similar to our previous report inasmuch as gamma activity is a marker for enhanced sensory awareness. The concomitant increased relative frontal theta power and decreased frontal delta power during meditation further support that this form of meditation involves increased baseline frontal activity with top-down control over frontal attentional capture due to environmental input and concomitantly enhanced sensory perceptivity. This view is in contrast to early definitions of meditation as a form of relaxation or sleep-like state, although it is important to note that the variety of very different meditative practices do include those with a greater similarity to sleep states (e.g., Yoga Nidra), which may be marked by opposite or markedly different findings from those reported here (Cahn and Polich 2006).
The current findings emphasize that in highly practiced Vipassana meditation practitioners, the primary effects of meditation state on brain rhythms are centered in the low (delta) and high (gamma) frequency ranges, with moderate relative increase in frontal theta, and gamma effects most pronounced in more advanced practitioners. Given the well-known association of increased slow delta activity during deep sleep and the more recently described decrease in gamma power during sleep (Cantero and Atienza 2005; Cantero et al. 2004; Maloney et al. 1997), the overall picture can be interpreted as a state of enhanced "awakeness." Further, alpha power does not reliably differentiate between meditation and control state in advanced Vipassana practitioners but instead tends to vary inversely with drowsiness. Mindfulness/open-monitoring practices that involve the widening of the attentional spotlight to presentmoment sensory experiences may be characterized by a mode of frontal engagement mediating enhanced stimulus representation and clarity of awareness (spontaneous gamma, evoked N1) and concomitant decreased cognitive elaboration upon stimuli in the environment (evoked P2, $\mathrm{P} 3 \mathrm{a})$. In contrast, practices involving the narrowing of the attentional spotlight such as mantra and breath-focused attention practices may likely be characterized by greater frontal midline theta engagement and less enhanced measures of stimulus representation, a hypothesis requiring further specific study of these two forms of practice using the same protocol across groups.

\section{Conclusions}

Appreciation for the variety of mental practices subsumed by the name "meditation" has recently become a salient research topic, as observation of the various types of attentional engagement across meditative practices may promote different neurophysiologic outcomes (Cahn and Polich 2006; Depraz et al. 2003; Dunn et al. 1999; Lehmann et al. 2001; Lutz et al. 2004, 2008). Assessment of this group of Vipassana meditators has previously demonstrated decreased frontal engagement in the processing of unexpected and aversive stimuli during meditation in the setting of a trend toward enhanced sensory representation of the standard and oddball stimuli as indexed by increased $\mathrm{N} 1$ amplitudes. The present findings are that of enhanced frontal engagement as indexed by decreased frontal delta power and relative increase in the frontal component of theta activity and a broad increase in parieto-occipital gamma activity during the meditative state prior to the onset of the tones used to elicit the findings in our earlier study. No other frequency bands reliably distinguished the two states.

It is theoretically plausible that the enhanced gamma activity observed in this dataset is related to the iterative 
body scanning aspect of the technique. Alternatively, this widespread posterior increase in gamma power may be more generally related to the enhanced perceptual clarity often reported in open-monitoring meditative states. Further studies contrasting this meditative practice with focused attention practices such as those involving breath awareness, mantra, and/or visualization would provide significant insight into the specificity of the delta, theta and gamma effects seen in these practitioners. An initial hypothesis would be that the focused attention practices might engage the frontal theta activity to a greater degree than open-monitoring practices such as Vipassana and less engagement of the gamma activity seen here. An alternative would be to observe more localized gamma increases to the cortical areas representing the object of attention. Of importance also for the development of the field is the relation between these meditation state changes in the brain and experiential qualities that must be assessed with improved psychometric analyses.

Acknowledgments This work was supported by NIH grants DA018262 and AA006420, The Fetzer Institute, the NIH Medical Scientist Training Grant T32 GM07198, and the NIH Postdoctoral grant T32 MH019934 grant in part supported BRC, who is also affiliated with the Laboratory for Psychopharmacology and Brain Imaging, University of Zurich Hospital of Psychiatry. The help and guidance of Drs. Mark Geyer and Franz Vollenweider are gratefully acknowledged. We thank the meditator participants and Mr. John Beary of Vipassana Research International for assistance in recruiting meditation participants. This paper is $\operatorname{xxxxxx}$ from The Scripps Research Institute.

Open Access This article is distributed under the terms of the Creative Commons Attribution Noncommercial License which permits any noncommercial use, distribution, and reproduction in any medium, provided the original author(s) and source are credited.

\section{References}

Aftanas LI, Golocheikine SA (2001) Human anterior and frontal midline theta and lower alpha reflect emotionally positive state and internalized attention: high-resolution EEG investigation of meditation. Neurosci Lett 310:57-60

Aftanas LI, Golocheikine SA (2003) Changes in cortical activity in altered states of consciousness: the study of meditation by highresolution EEG. Hum Physiol 29:143-151

Aftanas L, Golosheykin S (2005) Impact of regular meditation practice on EEG activity at rest and during evoked negative emotions. Int J Neurosci 115:893-909

Anand BK, Chhina GS, Singh B (1961a) Some aspects of electroencephalographic studies in yogis. Electroencephal Clin Neurophysiol 13:452-456

Anand BK, Chhina GS, Singh B (1961b) Studies on Shri Ramanand yogi during his stay in an air-tight box. Indian J Med Res 49:82-89

Babiloni C, Frisoni G, Steriade M, Bresciani L, Binetti G, Del Percio C, Geroldi C, Miniussi C, Nobili F, Rodriguez G, Zappasodi F, Carfagna T, Rossini PM (2006) Frontal white matter volume and delta EEG sources negatively correlate in awake subjects with mild cognitive impairment and Alzheimer's disease. Clin Neurophysiol 117:1113-1129

Baijal S, Srinivasan N (2009) Theta activity and meditative states: spectral changes during concentrative meditation. Cogn Process

Banquet JP (1973) Spectral analysis of the EEG in meditation. Electroencephalogr Clin Neurophysiol 35:143-151

Basar E, Basar-Eroglu C, Karakas S, Schurmann M (2001a) Gamma, alpha, delta, and theta oscillations govern cognitive processes. Int J Psychophysiol 39:241-248

Basar E, Schurmann M, Sakowitz O (2001b) The selectively distributed theta system: functions. Int $\mathrm{J}$ Psychophysiol 39:197-212

Basar-Eroglu C, Struber D, Schurmann M, Stadler M, Basar E (1996) Gamma-band responses in the brain: a short review of psychophysiological correlates and functional significance. Int $\mathrm{J}$ Psychophysiol 24:101-112

Benjamini Y, Yekutieli D (2001) The control of the false discovery rate in multiple testing under dependency. Ann Stat 29:11651188

Cahn BR, Polich J (2006) Meditation states and traits: EEG, ERP, and neuroimaging studies. Psychol Bull 132:180-211

Cahn BR, Polich J (2009) Meditation (Vipassana) and the P3a eventrelated brain potential. Int J Psychophysiol 72:51-60

Cantero JL, Atienza M (2005) The role of neural synchronization in the emergence of cognition across the wake-sleep cycle. Rev Neurosci 16:69-83

Cantero JL, Atienza M, Madsen JR, Stickgold R (2004) Gamma EEG dynamics in neocortex and hippocampus during human wakefulness and sleep. Neuroimage 22:1271-1280

Christoff K, Gordon AM, Smallwood J, Smith R, Schooler JW (2009) Experience sampling during fMRI reveals default network and executive system contributions to mind wandering. Proc Natl Acad Sci USA 106:8719-8724

Comon P (1994) Independent component analysis: a new concept? Signal Process 36:287-314

Darvas F, Pantazis D, Kucukaltun-Yildirim E, Leahy RM (2004) Mapping human brain function with MEG and EEG: methods and validation. Neuroimage 23(1):S289-S299

Das NN, Gastaut H (1957) Variations de l'activite electrique du cerveau, du coeur et des muscles squelettiques au cours de la meditation et de l'extase yogique. Electroencephalogr Clin Neurophysiol 6:211-219

Davidson RJ (2003) Affective neuroscience and psychophysiology: toward a synthesis. Psychophysiology 40:655-665

de Jongh A, de Munck JC, Baayen JC, Jonkman EJ, Heethaar RM, van Dijk BW (2001) The localization of spontaneous brain activity: first results in patients with cerebral tumors. Clin Neurophysiol 112:378-385

de Lange FP, Jensen O, Bauer M, Toni I (2008) Interactions between posterior gamma and frontal alpha/beta oscillations during imagined actions. Front Hum Neurosci 2:7

Delorme A (2006) Statistical methods. In: Webster JG (ed) Encyclopedia of medical device and instrumentation, vol 6. Wiley Interscience, Hoboken, NJ, USA, pp 240-264

Delorme A, Makeig S (2004) EEGLAB: an open source toolbox for analysis of single-trial EEG dynamics including independent component analysis. J Neurosci Methods 134:9-21

Delorme A, Sejnowski T, Makeig S (2007) Enhanced detection of artifacts in EEG data using higher-order statistics and independent component analysis. Neuroimage 34:1443-1449

Depraz N, Varela JF, Vermersch P (2003) On becoming aware: a pragmatics of experiencing. John Benjamins Publishing Company, Amsterdam

Dimigen O, Valsecchi M, Sommer W, Kliegl R (2009) Human microsaccade-related visual brain responses. J Neurosci 29:12321-12331 
Dunn BR, Hartigan JA, Mikulas WL (1999) Concentration and mindfulness meditations: unique forms of consciousness? Appl Psychophysiol Biofeedback 24:147-165

Faber PL, Steiner ME, Lehmann D, Pascual-Marqui RD, Jancke L, Esslen M, Gianotti LRR (2008) Deactivation of the medial prefrontal cortex in experienced Zen meditators (Abstract). Brain Topogr 20:172

Fell J, Fernandez G, Klaver P, Elger CE, Fries P (2003) Is synchronized neuronal gamma activity relevant for selective attention? Brain Res Brain Res Rev 42:265-272

Fries P, Neuenschwander S, Engel AK, Goebel R, Singer W (2001) Rapid feature selective neuronal synchronization through correlated latency shifting. Nat Neurosci 4:194-200

Fries P, Womelsdorf T, Oostenveld R, Desimone R (2008) The effects of visual stimulation and selective visual attention on rhythmic neuronal synchronization in macaque area V4. J Neurosci 28:4823-4835

Gaylord C, Orme-Johnson D, Travis F (1989) The effects of the transcendental meditation technique and progressive muscle relaxation on EEG coherence, stress reactivity, and mental health in black adults. Int J Neurosci 46:77-86

Gross J, Schnitzler A, Timmermann L, Ploner M (2007) Gamma oscillations in human primary somatosensory cortex reflect pain perception. PLoS Biol 5:e133

Grossman P, Niemann L, Schmidt S, Walach H (2004) Mindfulnessbased stress reduction and health benefits. A meta-analysis. J Psychosom Res 57:35-43

Gunaratana H (2002) Mindfulness in plain English. Wisdom Publications, Boston, MA

Hart W (1987) The art of living: Vipassana meditation as taught by Goenka SN New York: HarperOne

Hebert R, Lehmann D (1977) Theta bursts: an EEG pattern in normal subjects practising the transcendental meditation technique. Electroencephalogr Clin Neurophysiol 42:397-405

Hyvärinen A, Oja E (2000) Independent component analysis: algorithms and application. Neural Netw 13:411-430

Jung TP, Makeig S, Humphries C, Lee TW, McKeown MJ, Iragui V, Sejnowski TJ (2000a) Removing electroencephalographic artifacts by blind source separation. Psychophysiology 37:163-178

Jung TP, Makeig S, Westerfield M, Townsend J, Courchesne E, Sejnowski TJ (2000b) Removal of eye activity artifacts from visual event-related potentials in normal and clinical subjects. Clin Neurophysiol 111:1745-1758

Kabat-Zinn J (1982) An outpatient program in behavioral medicine for chronic pain patients based on the practice of mindfulness meditation: theoretical considerations and preliminary results. Gen Hosp Psychiatry 4:33-47

Kabat-Zinn J (2003) Mindfulness-based interventions in context: past, present, and future. Clin Psychol Sci Pract 10:144-158

Kasamatsu A, Hirai T (1966) An electroencephalographic study on the Zen meditation (Zazen). Folia Psychiatr Neurol Jpn 20:315336

Kasamatsu A, Okuma T, Takenaka S, Koga E, Ikada K, Sugiyama H (1957) The EEG of 'Zen' and 'Yoga' practitioners. Electroencephalogr Clin Neurophysiol 9:51-52

Kinoshita K (1975) A study on response of EEG during Zen meditation-alpha-blocking to name calling (in Japanese). Seishin Shinkeigaku Zasshi 77:623-658

Lachaux JP, George N, Tallon-Baudry C, Martinerie J, Hugueville L, Minotti L, Kahane P, Renault B (2005) The many faces of the gamma band response to complex visual stimuli. Neuroimage 25:491-501

Lehmann D, Faber PL, Achermann P, Jeanmonod D, Gianotti LR, Pizzagalli D (2001) Brain sources of EEG gamma frequency during volitionally meditation-induced, altered states of consciousness, and experience of the self. Psychiatry Res 108:111-121
Lehrer PM, Schoicket S, Carrington P, Woolfolk RL (1980) Psychophysiological and cognitive responses to stressful stimuli in subjects practicing progressive relaxation and clinically standardized meditation. Behav Res Ther 18:293-303

Lutz A, Greischar LL, Rawlings NB, Ricard M, Davidson RJ (2004) Long-term meditators self-induce high-amplitude gamma synchrony during mental practice. Proc Natl Acad Sci USA 101:16369-16373

Lutz A, Dunne JD, Davidson RJ (2007) Meditation and the neuroscience of consciousness. In: Zelazo PD, Moscovitch M, Thompson E (eds) The Cambridge handbook of consciousness. Cambridge University Press, Cambridge, UK

Lutz A, Slagter HA, Dunne JD, Davidson RJ (2008) Attention regulation and monitoring in meditation. Trends Cogn Sci 12:163-169

Lutz A, Slagter HA, Rawlings NB, Francis AD, Greischar LL, Davidson RJ (2009) Mental training enhances attentional stability: neural and behavioral evidence. J Neurosci 29:13418-13427

Maloney KJ, Cape EG, Gotman J, Jones BE (1997) High-frequency gamma electroencephalogram activity in association with sleepwake states and spontaneous behaviors in the rat. Neuroscience 76:541-555

Meador KJ, Ray PG, Echauz JR, Loring DW, Vachtsevanos GJ (2002) Gamma coherence and conscious perception. Neurology $59: 847-854$

Melloni L, Molina C, Pena M, Torres D, Singer W, Rodriguez E (2007) Synchronization of neural activity across cortical areas correlates with conscious perception. J Neurosci 27:2858-2865

Mitchell DJ, McNaughton N, Flanagan D, Kirk IJ (2008) Frontalmidline theta from the perspective of hippocampal "theta". Prog Neurobiol 86:156-185

Niedermeyer E (1997) Alpha rhythms as physiological and abnormal phenomena. Int J Psychophysiol 26:31-49

Niedermeyer E, Lopes da Silva FH (1999) Electroencephalography: basic principles, clinical applications and related fields. Baltimore: Williams and Wilkins

Ott U (2001) The EEG and the depth of meditation. J Med Med Res 1:55-68

Pagnoni G, Cekic M, Guo Y (2008) "Thinking about not-thinking": neural correlates of conceptual processing during Zen meditation. PLoS One 3:e3083

Pan W, Zhang L, Xia Y (1994) The difference in EEG theta waves between concentrative and non-concentrative qigong states-a power spectrum and topographic mapping study. J Tradit Chin Med 14:212-218

Penolazzi B, Spironelli C, Angrilli A (2008) Delta EEG activity as a marker of dysfunctional linguistic processing in developmental dyslexia. Psychophysiology 45:1025-1033

Polich J (2007) Updating P300: an integrative theory of P3a and P3b. Clin Neurophysiol 118:2128-2148

Polich J, Kok A (1995) Cognitive and biological determinants of P300: an integrative review. Biol Psychol 41:103-146

Rabiner LR, Gold B (1975) Theory and application of digital signal processing. Prentice-Hall, Englewood Cliffs, NJ

Reva NV, Aftanas LI (2004) The coincidence between late nonphase-locked gamma synchronization response and saccadic eye movements. Int J Psychophysiol 51:215-222

Rodriguez E, George N, Lachaux JP, Martinerie J, Renault B, Varela FJ (1999) Perception's shadow: long-distance synchronization of human brain activity. Nature 397:430-433

Sauve K (1999) Gamma-band synchronous oscillations: recent evidence regarding their functional significance. Conscious Cogn 8:213-224

Segal ZV, Williams JMG, Teasdale JD (2002) Mindfulness-based cognitive therapy for depression: a new approach to preventing relapse. Guilford Press, New York 
Singer W (1993) Synchronization of cortical activity and its putative role in information processing and learning. Annu Rev Physiol 55:349-374

Slagter HA, Lutz A, Greischar LL, Francis AD, Nieuwenhuis S, Davis JM, Davidson RJ (2007) Mental training affects distribution of limited brain resources. PLoS Biol 5:1228-1235

Slagter HA, Lutz A, Greischar LL, Nieuwenhuis S, Davidson RJ (2009) Theta phase synchrony and conscious target perception: impact of intensive mental training. J Cogn Neurosci 21:15361549

Smallwood J, Schooler JW (2006) The restless mind. Psychol Bull 132:946-958

Smallwood J, McSpadden M, Schooler JW (2007) The lights are on but no one's home: meta-awareness and the decoupling of attention when the mind wanders. Psychon Bull Rev 14:527-533

Smallwood J, Beach E, Schooler JW, Handy TC (2008) Going AWOL in the brain: mind wandering reduces cortical analysis of external events. J Cogn Neurosci 20:458-469

Tei S, Faber PL, Lehmann D, Tsujiuchi T, Kumano H, PascualMarqui RD, Gianotti LR, Kochi K (2009) Meditators and nonmeditators: EEG source imaging during resting. Brain Topogr 22:158-165

Thomson DJ (1982) Spectrum estimation and harmonic analysis. Proceed IEEE 70:1055-1096
Travis F (1991) Eyes open and TM EEG patterns after one and eight years of TM practice. Psychophysiology 28:58

Travis F, Pearson C (1999) Pure consciousness: distinct phenomenological and physiological correlates of "consciousness itself". Int J Neurosci 100:77-89

Travis F, Tecce J, Arenander A, Wallace RK (2002) Patterns of EEG coherence, power, and contingent negative variation characterize the integration of transcendental and waking states. Biol Psychol 61:293-319

Varela FJ (1995) Resonant cell assemblies: a new approach to cognitive functions and neuronal synchrony. Biol Res 28:81-95

Wallace RK (1970) Physiological effects of transcendental meditation. Science 167:1751-1754

Wenger MA, Bagchi BK (1961) Studies of autonomic functions in practitioners of yoga in India. Behav Sci 6:312-323

Wilcox RR (2005) Introduction to Robust estimation and hypothesis testing, 2nd edn. Elsevier Academic Press, San Diego, CA, USA

Yuval-Greenberg S, Deouell LY (2009) The broadband-transient induced gamma-band response in scalp EEG reflects the execution of saccades. Brain Topogr 22:3-6

Yuval-Greenberg S, Tomer O, Keren AS, Nelken I, Deouell LY (2008) Transient induced gamma-band response in EEG as a manifestation of miniature saccades. Neuron 58:429-441 